\title{
Thermal Expansion of Supported and Freestanding Graphene: Lattice Constant versus Interatomic Distance
}

\author{
Monica Pozzo, ${ }^{1}$ Dario Alfê,,${ }^{1,2}$ Paolo Lacovig, ${ }^{3}$ Philip Hofmann, ${ }^{4}$ Silvano Lizzit, ${ }^{3}$ and Alessandro Baraldi ${ }^{5,6, *}$ \\ ${ }^{1}$ Department of Earth Sciences, Department of Physics and Astronomy, TYC@UCL, and London Centre for Nanotechnology, \\ University College London, Gower Street, London WC1E 6BT, United Kingdom \\ ${ }^{2}$ IOM-CNR, DEMOCRITOS National Simulation Centre, I-34100 Trieste, Italy \\ ${ }^{3}$ Sincrotrone Trieste S.C.p.A., Area Science Park, S.S. 14 Km 163.5, I-34149 Trieste, Italy \\ ${ }^{4}$ Department of Physics and Astronomy and Interdisciplinary Nanoscience Center (iNANO), Aarhus University, \\ 8000 Aarhus C, Denmark \\ ${ }^{5}$ Physics Department and CENMAT, University of Trieste, Via Valerio 2, I-34127 Trieste, Italy \\ ${ }^{6}$ IOM-CNR, Laboratorio TASC, Area Science Park, S.S. 14 Km 163.5, I-34149 Trieste, Italy
}

(Received 30 September 2010; published 29 March 2011)

\begin{abstract}
By using ab initio molecular dynamics calculations, we show that even where the graphene lattice constant contracts, as previously reported for freestanding graphene below room temperature, the average carbon-carbon distance increases with temperature, in both free and supported graphene. This results in a larger corrugation at higher temperature, which can affect the interaction between graphene and the supporting substrate. For a weakly interacting system as graphene/ $\operatorname{Ir}(111)$, we confirm the results using an experimental approach which gives direct access to interatomic distances.
\end{abstract}

DOI: 10.1103/PhysRevLett.106.135501

PACS numbers: 81.05.ue, 68.65.-k, 65.80.Ck

Graphene's (GR) mechanical [1] and thermal [2,3] properties are crucial for applications such as the cooling of electronic devices $[4,5]$, but their microscopic mechanism is often not well understood [6], in contrast to that of many electronic phenomena [7-10]. A particular challenge lies in the fact that graphene, while being two dimensional, exists in a three-dimensional world, permitting low-lying vibrational excitations and the formation of large-scale ripples perpendicular to the plane $[11,12]$. This is also important because it introduces a difference between freestanding GR and the technologically more important supported material.

In particular, the thermal expansion coefficient of GR and the underlying microscopic mechanism is currently being debated. For freestanding GR and below $\approx 500-700 \mathrm{~K}$, a state-of-the-art atomistic simulation [13], a nonequilibrium Green's function approach [14], and a harmonic density functional theory (HDFT) calculation $[15,16]$ all show a decreasing in-plane lattice parameter $a$, i.e., a thermal contraction. Above $900 \mathrm{~K}$ or so, the two former techniques show a trend reversal with a thermal expansion, while in HDFT the contraction persists over the entire temperature range studied (up to $2500 \mathrm{~K}$ ). Experiments clearly confirm the thermal contraction of $a$ below room temperature $[3,17]$, but no high temperature measurements have been reported.

In order to clarify this issue and to gain insight into its microscopic origin, we present a study based on $a b$ initio simulations and core level photoelectron spectroscopy. Temperature-dependent $a b$ initio molecular dynamics (AIMD) calculations were performed on both freestanding and supported GR. We have used the VASP code [18] with the projector-augmented wave method $[19,20]$, the Perdew-Burke-Ernzerhof exchange-correlation energy [21], and an efficient extrapolation for the charge density [22]. Single particle orbitals were expanded in plane waves with a cutoff of $400 \mathrm{eV}$. We used the NPT ensemble (constant particles number $N$, pressure $P$, and temperature $T)$, as recently implemented in VASP $[23,24]$. For the present slab calculations, we only applied the constant pressure algorithm to the two lattice vectors parallel to the surface, leaving the third unchanged during the simulation.

In the case of freestanding GR the calculations were done using unit cells of different sizes $(8 \times 8,10 \times 10$, and $16 \times 16$, corresponding to 128,200 , and $512 \mathrm{C}$ atoms, respectively) and temperatures between 0 and $2000 \mathrm{~K}$. AIMD simulations were performed at the $\Gamma$ point, each simulation lasting over 50 ps. The average distance $d$ between the $\mathrm{C}$ atoms was obtained by integrating the first peak of the radial distribution function. The average in-plane lattice constant $a$ was obtained by time averaging the length of the two lattice parameters in the plane. At each temperature we also extracted 20 statistically independent configurations and calculated the $\mathrm{C} 1 s$ core electron binding energy (BE), including final state effects, of each of the $200 \mathrm{C}$ atoms in the cell, for a total of 4000 configurations [25]. For supported GR the adsorption has been modeled by overlaying a $10 \times 10$ GR sheet $(200 \mathrm{C}$ atoms) over a $9 \times 9 \operatorname{Ir}(111)$ supercell [26] and using a slab of $3 \mathrm{Ir}$ layers where the two topmost layers were allowed to move while the bottom layer was held fixed. In this case the simulations were performed between 300 and $2000 \mathrm{~K}$ and lasted about 40 ps. 
Figure 1 displays the results of our AIMD simulation for the average nearest-neighbor distance $d$ [Fig. 1(a)] and the average in-plane lattice constant $a$ [Fig. 1(b)] for both supported and freestanding GR. It is important to note that $a$ is not necessarily a simple function of $d(a=\sqrt{3} d$ for GR) because of out-of-plane vibrations and ripples.

The results show that for both freestanding and supported GR, $d$ increases with temperature; i.e., it shows the usual behavior for a solid. The rate of expansion does not depend on the simulation cell size, as verified for freestanding GR modeled using different number of $\mathrm{C}$ atoms $n$. The emerging picture for $a$ is quite different for the free and the supported GR case. For freestanding GR a thermal contraction of $a$ is found for all simulation cell sizes at lower temperatures, in qualitative agreement with previous calculations $[13,14,16]$ and experiments $[3,17]$. For temperatures higher than $1000 \mathrm{~K}, a$ slightly increases for the two smaller simulation cells while it decreases for the larger one. This dependence of $a$ on the size of the system is consistent with the existence of large-scale ripples $[11,12]$ which cannot be fitted inside the relatively small simulation cells. By contrast, the prediction for $d$ is
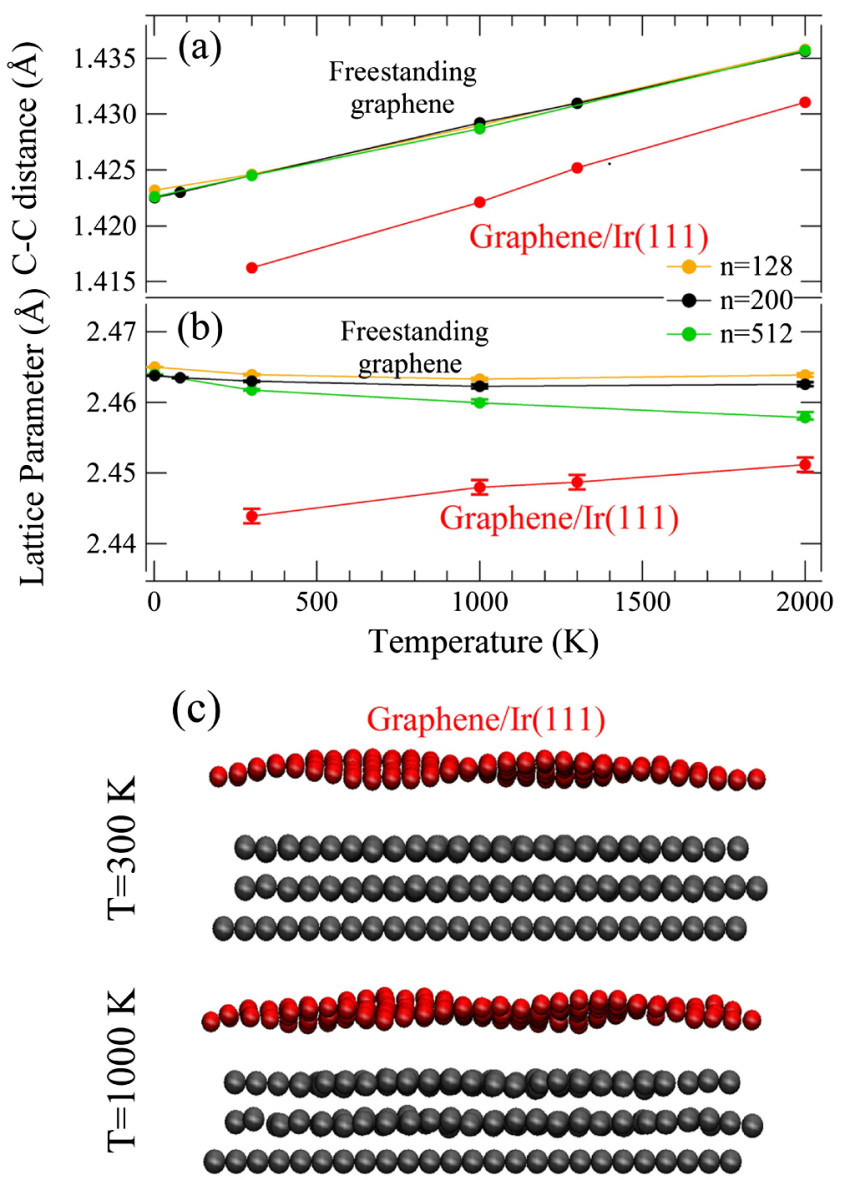

FIG. 1 (color online). Temperature dependence of the (a) average C-C distances and (b) lattice parameter for supported GR on $\operatorname{Ir}(111)$ and freestanding GR. (c) Simulation snapshots of the GR/Ir(111) system at 300 and $1000 \mathrm{~K}$. well converged even with the smallest simulation cell. The thermal contraction of $a$ in freestanding GR has been explained by the negative Grüneisen parameter of lowlying phonon modes, i.e., a membrane effect [16].

Our results for supported GR resting on an $\operatorname{Ir}(111)$ surface show that the variation of $a$ with temperature is positive and mainly governed by the Ir slab. We also observe that, while the short-range corrugation of the GR layer increases with temperature, as expected, the amplitude of the long-range fluctuations is preserved [snapshots displayed in Fig. 1(c)]. Remarkably, the temperature dependence of $d\left(\Delta d / d=8.7 \times 10^{-6} \mathrm{~K}^{-1}\right)$ is similar to the one found for the freestanding GR layer $\left(6.5 \times 10^{-6} \mathrm{~K}^{-1}\right)$, despite the largely different temperature dependence of the lattice parameter $a$ (positive expansion in the former, negative in the latter).

As the simulations clearly produce consistent results for $d$, a direct experimental test of this value is desirable. It is important to underline that most experiments, such as macroscopic deformation or diffraction, yield $a$ rather than $d$. In order to overcome this limitation, here we have measured the temperature dependence of the $\mathrm{C} 1 s$ signal, using high-energy resolution $\mathrm{X}$-ray photoelectron spectroscopy (XPS) and taking into account the band-dispersion properties of this core state [27].

The experiments were performed at the SuperESCA beam line of Elettra. The GR layer was prepared on $\operatorname{Ir}(111)$ using a well-established procedure [28]. C $1 s$ core level spectra were acquired at a photon energy of $400 \mathrm{eV}$ with a Phoibos electron energy analyzer, implemented with a homemade delay-line detection system which allows us to measure each photoemission spectrum in $500 \mathrm{~ms}$. Spectra were taken during cooling of the GR/Ir system, as to avoid any effect of the heating on the measurements, permitting an accurate determination of $\mathrm{BE}$ and line shape of the single component $\mathrm{C} 1 s$ spectrum in a large temperature range [Fig. 2(a)].

The C $1 s$ spectra were fitted by convoluting a DoniachSunjić (DS) function with a Gaussian which accounts for phonon, inhomogeneous, and instrumental broadening. The DS profile contains a Lorentzian distribution arising from the finite core-hole lifetime and an asymmetry parameter for electron-hole pair excitations. Intensity, BE, and asymmetry were left as free parameters, while the Lorentzian width was held at $125 \mathrm{meV}$, as obtained for the low temperature spectrum. The analysis reveals that the single C $1 s$ component, characteristic of the GR/Ir(111) system [26], moves to higher BEs [gray curve in Fig. 2(b)] and broadens with increasing temperature.

In order to obtain information about $d$, we have measured the bonding-antibonding splitting $\Delta E$ in the carbon $1 s$ core state [27]. In a simple tight-binding picture, the size of $\Delta E$ would be expected to decrease exponentially with the C-C distance $d . \Delta E$ can be measured by taking data at the center of two different surface Brillouin zones, such 

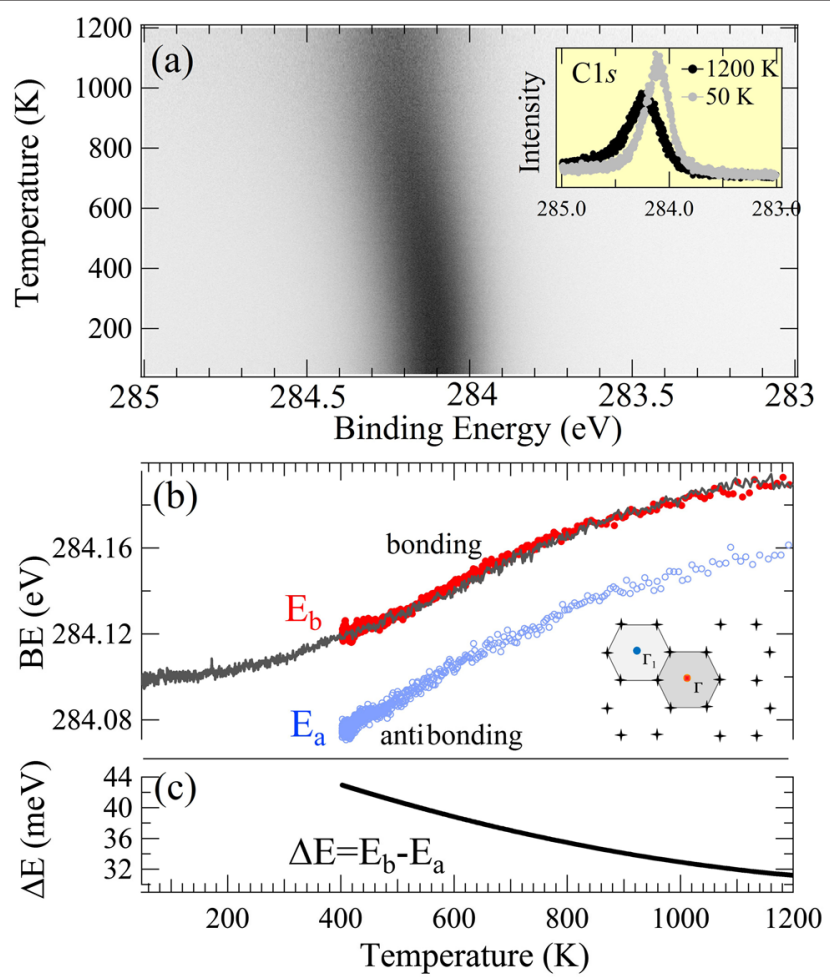

FIG. 2 (color online). (a) Temperature-dependent C $1 s$ normal emission (1470 spectra) taken shown as a two-dimensional intensity plot. Inset: Spectra measured at the highest and the lowest temperature. (b) Temperature dependence of the $\mathrm{C} 1 s \mathrm{BE}$. The gray curve is obtained from the data set shown in (a). Red $\left[\left(E_{b}\right)\right.$, filled circles] and blue $\left[\left(E_{a}\right)\right.$, empty circles] curves correspond to the bonding and antibonding states, respectively. (c) $\mathrm{BE}$ difference between the bonding and the antibonding component.

that the antibonding contribution $\left(E_{a}\right)$ is suppressed in one data set and the bonding contribution $\left(E_{b}\right)$ in the other. However, the determination of the temperature-dependent splitting between the bonding and antibonding components could not be done with the same sample manipulator used for the extended temperature range, since it does not have the required sample alignment capabilities. Using a different manipulator, a second set of data was measured in the same way, but over a more limited temperature range. Data shown in Fig. 2(b) were taken at normal emission (bonding component $E_{b}$ ) and emission from the center of the neighboring Brillouin zone (antibonding component $E_{a}$ ). The data for the bonding component measured with the two different manipulators agree very well within their overlapping temperature range. Figure $2(\mathrm{c})$ gives the BE difference $\Delta E(T)=E_{b}(T)-E_{a}(T)$, obtained as the difference between polynomial fits of the data points $E_{b}$ and $E_{a}$. In the observed temperature range of $\approx 800 \mathrm{~K}, \Delta E$ decreases from $44 \pm 2$ to $32 \pm 2 \mathrm{meV}$, strongly supporting an increase of $d$. In fact, $\Delta E(T)$ can be fitted well with an exponential, suggesting a linear thermal expansion of $d$ in the whole temperature range.
This result and the validity of our $a b$ initio calculations become even more evident if we compare the change in splitting with the overall picture for the $\mathrm{C} 1 \mathrm{~s}$ core level splitting in $\mathrm{C}-\mathrm{C}$ bonds. Figure 3 shows this splitting over a wide range of $d$, using data points for $\mathrm{C}_{2} \mathrm{H}_{2}$ [29], $\mathrm{C}_{2} \mathrm{H}_{4}$ [30], as well as our results for 400 and $1000 \mathrm{~K}$. These latter points have been plotted such that the size of their splitting is taken from the experiment while the corresponding value of $d$ is that of the calculation. Clearly, the two points fit very well into the overall picture, confirming our ability to compute $d$ reliably for this system.

Further information on GR's thermodynamic properties can be extracted from the thermal broadening of the XPS spectra. The experimentally determined Gaussian width shown in Fig. 4(a) (gray dots) exhibits the expected increase with temperature. These experimental results have been compared with the calculated statistical distribution of the C $1 s$ BEs reported in Fig. 4(b). The strong thermal broadening is evident for both the supported and the freestanding GR. The agreement is clearly excellent for the supported layer, indicating again that our calculational approach gives the correct statistical distribution of the positions of the $\mathrm{C}$ atoms.

The behavior of the experimental Gaussian width has been analyzed using the Hedin-Rosengren theory for the temperature-induced phonon broadening [31] [Fig. 4(a), black curve]. We found a GR Debye temperature of $1495 \pm 50 \mathrm{~K}$, between the values of diamond $(2240 \mathrm{~K})$ and graphite (402 K) [32]. This is in good agreement with the calculated out-of-plane Debye temperature of GR (1287 K) [33], thus suggesting that the out-of-plane atomic fluctuations, as found in the simulations [see Fig. 1(c)], play an important role.

We finally compare the calculated and the measured $\mathrm{C}$ $1 s \mathrm{BE}$ values. Since only temperature-induced relative shifts are meaningful, we take the $\mathrm{C} 1 s \mathrm{BE}$ at $300 \mathrm{~K}$ as a reference point. The calculated shift is qualitatively different for the freestanding and the supported GR. The former shows quite a large shift to smaller BEs as the temperature is raised [Fig. 4(b), bottom]. This behavior is what would be obtained in an experiment performed on a freestanding

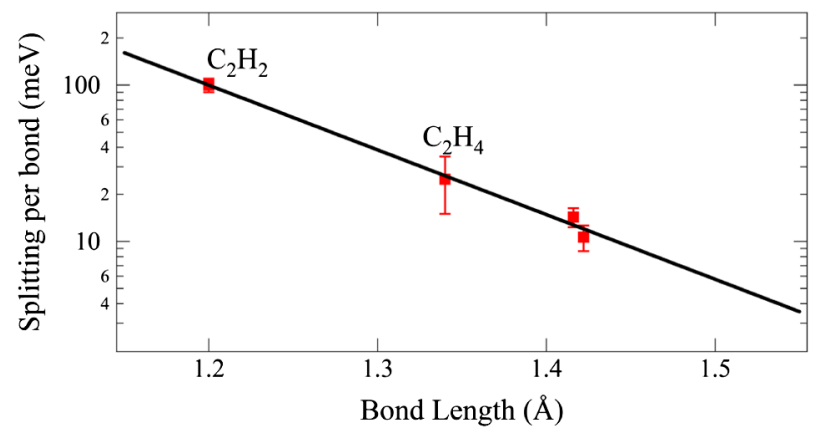

FIG. 3 (color online). C $1 s$ splitting in $\mathrm{C}-\mathrm{C}$ bonds as a function of bond length. The values for $\mathrm{C}_{2} \mathrm{H}_{2}$ and $\mathrm{C}_{2} \mathrm{H}_{4}$ come from Refs. [29,30]. 


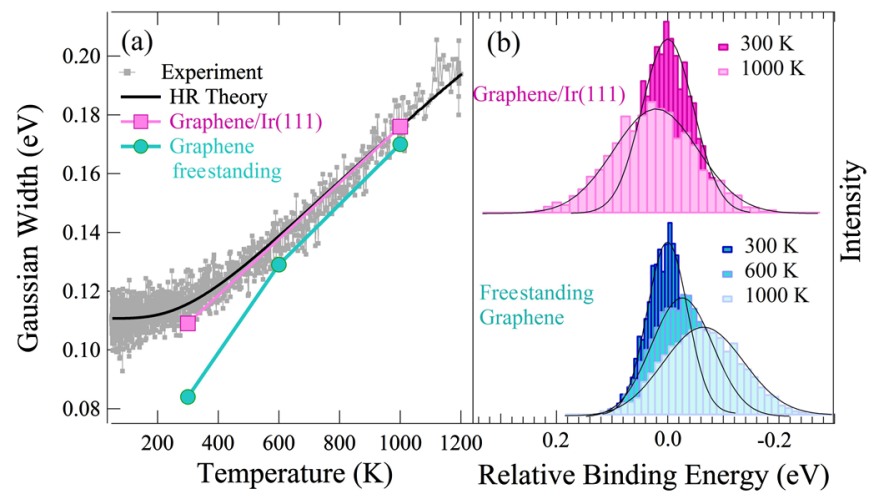

FIG. 4 (color online). (a) Temperature-dependent Gaussian width of the $\mathrm{C} 1 s$ spectra. The gray small markers are experimental data (after subtraction of the instrumental, inhomogeneous, and zero-temperature broadening contributions), the black line is a fit within Hedin-Rosengren (HR) model for phonon-induced broadening. The blue (circles) and pink (squares) curves represent the Gaussian width of the calculated statistical distribution of BEs. (b) Calculated statistical distribution of BEs for freestanding and supported GR.

GR layer. By contrast, for the Ir supporting the GR sheet the shift is towards higher BEs [Fig. 4(b), top]. This is in qualitative agreement with the experiment even though it is somewhat smaller $(20 \mathrm{meV}$ rather than $70 \mathrm{meV}$ between 300 and $1000 \mathrm{~K}$ ).

In conclusion, we have shown that the $\mathrm{C}-\mathrm{C}$ nearestneighbor distance increases in all cases studied, even when the lattice constant contracts. In addition to improving the understanding of GR's properties at a fundamental level, our results have important implications for nanotechnological applications of supported GR. The temperature dependence of the $\mathrm{C}-\mathrm{C}$ distance is directly related to phonon-substrate interactions and represents a key factor for understanding the problems of heat dissipation and for the control of thermally induced stress when GR is used as a material for cooling nanoscale electronic devices $[4,5]$ or as the device material as such.

The work of D. A. and M. P. was conducted as part of a EURYI grant as provided by EPSRC. Calculations were performed on the HECToR national service (UK) and on JaguarPF at the National Center for Computational Science (U.S.). We acknowledge financial support by the Royal Society and the Danish Council for Independent Research. *alessandro.baraldi@elettra.trieste.it

[1] C. Lee et al., Science 321, 385 (2008).

[2] A. A. Balandin et al., Nano Lett. 8, 902 (2008).

[3] W. Bao et al., Nature Nanotech. 4, 562 (2009).

[4] R. Prasher, Science 328, 185 (2010).

[5] J. H. Seol et al., Science 328, 213 (2010).

[6] A. K. Geim, Science 324, 1530 (2009).

[7] K.S. Novoselov et al., Nature (London) 438, 197 (2005).

[8] Y. B. Zhang et al., Nature (London) 438, 201 (2005).

[9] A.H. Castro Neto et al., Rev. Mod. Phys. 81, 109 (2009).

[10] R. Balog et al., Nature Mater. 9, 315 (2010).

[11] J. C. Meyer et al., Nature (London) 446, 60 (2007).

[12] A. Fasolino, J. H. Los, and M. I. Katsnelson, Nature Mater. 6, 858 (2007).

[13] K. V. Zakharchenko, M. I. Katsnelson, and A. Fasolino, Phys. Rev. Lett. 102, 046808 (2009).

[14] J.-Wu Jiang, J.S. Wang, and B. Li, Phys. Rev. B 80, 205429 (2009).

[15] N. Bonini, M. Lazzeri, N. Marzari, and F. Mauri, Phys. Rev. Lett. 99, 176802 (2007).

[16] N. Mounet and N. Marzari, Phys. Rev. B 71, 205214 (2005).

[17] V. Singh et al., Nanotechnology 21, 165204 (2010).

[18] G. Kresse and J. Furthmüller, Phys. Rev. B 54, 11169 (1996).

[19] P. E. Blöchl, Phys. Rev. B 50, 17953 (1994).

[20] G. Kresse and D. Joubert, Phys. Rev. B 59, 1758 (1999).

[21] J. P. Perdew, K. Burke, and M. Ernzerhof, Phys. Rev. Lett. 77, 3865 (1996).

[22] D. Alfè, Comput. Phys. Commun. 118, 31 (1999).

[23] E. R. Hernández, A. Rodriguez-Prieto, A. Bergara, and D. Alfè, Phys. Rev. Lett. 104, 185701 (2010).

[24] E. Hernández, J. Chem. Phys. 115, 10282 (2001).

[25] We found that in order to obtain absolute convergence of the BEs to within $5 \mathrm{meV}$ we needed a $2 \times 2 \times 1 \mathbf{k}$-point grid.

[26] P. Lacovig et al., Phys. Rev. Lett. 103, 166101 (2009).

[27] S. Lizzit et al., Nature Phys. 6, 345 (2010).

[28] J. Coraux et al., New J. Phys. 11, 023006 (2009).

[29] B. Kempgens et al., Phys. Rev. Lett. 79, 3617 (1997).

[30] H. Koppel et al., J. Chem. Phys. 106, 4415 (1997).

[31] L. Hedin and A. Rosengren, J. Phys. F 7, 1339 (1977).

[32] A. K. Gschneidner, Jr., in Solid State Physics, edited by F. Seitz and D. Turnbull (Academic, New York, 1964), Vol. 16.

[33] V. K. Tewary and B. Yang, Phys. Rev. B 79, 125416 (2009). 